\title{
Valley-selective chiral phonon replicas of dark excitons and trions in monolayer WSe
}

\author{
Erfu Liu $\odot,{ }^{1}$ Jeremiah van Baren, ${ }^{1}$ Takashi Taniguchi, ${ }^{2}$ Kenji Watanabe, ${ }^{2}$ Yia-Chung Chang, ${ }^{3}$ and Chun Hung Lui ${ }^{1, *}$ \\ ${ }^{1}$ Department of Physics and Astronomy, University of California, Riverside, California 92521, USA \\ ${ }^{2}$ National Institute for Materials Science, Tsukuba, Ibaraki 305-004, Japan \\ ${ }^{3}$ Research Center for Applied Sciences, Academia Sinica, Taipei 11529, Taiwan
}

(Received 5 June 2019; published 25 October 2019)

\begin{abstract}
We observe a set of three replica luminescent peaks at $\sim 21.4 \mathrm{meV}$ below the dark exciton, and negative and positive dark trions (or exciton polarons) in monolayer $\mathrm{WSe}_{2}$. The replica redshift energy matches the energy of the zone-center $E^{\prime \prime}$-mode optical phonons. The phonon replicas exhibit a parallel gate dependence and the same $g$-factors as the dark excitonic states, but follow the valley selection rules of the bright excitonic states. While the dark states exhibit an out-of-plane transition dipole and valley-independent linearly polarized emission in the in-plane directions, their phonon replicas exhibit an in-plane transition dipole and valley-dependent circularly polarized emission in the out-of-plane directions. Our results and symmetry analysis show that the $K$-valley dark exciton decays into a left-handed chiral phonon and a right-handed photon, whereas the $K^{\prime}$-valley dark exciton decays into a right-handed chiral phonon and a left-handed photon. Such valley selection rules of chiral phonon replicas can be utilized to identify the valleys of the dark excitonic states and explore their chiral interactions with phonons.
\end{abstract}

DOI: 10.1103/PhysRevResearch.1.032007

Monolayer transition metal dichalcogenides (TMDs), such as $\mathrm{MoS}_{2}$ and $\mathrm{WSe}_{2}$, are two-dimensional (2D) semiconductors with remarkable excitonic properties [1-3]. They host tightly bound excitons at two time-reversal valleys $\left(K, K^{\prime}\right)$, where spin-orbit coupling splits each band into two subbands with opposite spins [4-14]. If the electrons and holes come from bands with the same electron spin, they form bright excitons with a short lifetime $(<10 \mathrm{ps})$ and relatively weak valley polarization due to the intervalley exchange interaction [Fig. 1(a)] [15]. But if the electrons and holes come from bands with opposite spins, they form dark excitons with a long lifetime ( $>100 \mathrm{ps}$ ) and relatively strong valley polarization due to the suppression of radiative recombination and intervalley exchange interactions [Fig. 1(b)] [16,17]. These excitons can further couple to the Fermi sea to form trions $[14,18]$ or exciton polarons [19-21] (hereafter we denote all of them as "trions" for simplicity). In monolayer $\mathrm{WS}_{2}$ and $\mathrm{WSe}_{2}$, the dark excitonic states can accumulate a large population because their energy level lies below the bright excitonic level [16,17,22-31]. These distinctive properties make the TMD dark excitonic states much better candidates than bright excitonic states for exciton transport, condensation, and valleytronic applications [32-34].

Detecting and manipulating the valley pseudospin of the dark excitonic states is, however, challenging because the usual valley selection rules of bright excitons are not

\footnotetext{
*Corresponding author: joshua.lui@ucr.edu

Published by the American Physical Society under the terms of the Creative Commons Attribution 4.0 International license. Further distribution of this work must maintain attribution to the author(s) and the published article's title, journal citation, and DOI.
}

applicable for dark excitons. The bright excitons exhibit inplane (IP) transition dipoles and couple selectively to light with right-handed (left-handed) circular polarization in the $K\left(K^{\prime}\right)$ valley [Fig. 1(a)] [35-38]. We can therefore conveniently access their valley pseudospin by light helicity. But the dark excitonic states exhibit out-of-plane (OP) transition dipoles and couple to vertically polarized light for both valleys [Fig. 1(b)] [17,22-31]. The lack of valley selection rules poses a great challenge to study the dark-state valley dynamics. In the search for new valley selection rules, the high-order processes, such as the decay of a dark exciton into a photon and a phonon, may exhibit valley selectivity. Li et al. recently reported a phonon replica of neutral dark exciton in monolayer $\mathrm{WSe}_{2}$ [39], but their results are insufficient to establish rigorous valley selection rules for dark excitons and trions.

In this Rapid Communication, we experimentally establish the valley and chirality selection rules for both dark excitons and trions in their chiral phonon replica emission in monolayer $\mathrm{WSe}_{2}$. Our experiment reveals a set of three replica luminescent peaks at $\sim 21.4 \mathrm{meV}$ below the dark exciton, and negative and positive dark trions. The redshift energy $(\sim 21.4 \mathrm{meV})$ matches the energy of the zone-center $E^{\prime \prime}$-mode optical phonons in monolayer $\mathrm{WSe}_{2}$ [40-42]. The replica emission exhibits a parallel gate dependence and the same $g$-factors as the original dark states, but follows distinct optical selection rules. While the original dark states exhibit OP dipoles and valley-independent linearly polarized emission in the IP directions, their phonon replicas exhibit IP dipoles and valley-dependent circularly polarized emission in the OP directions, similar to the characteristics of the bright excitonic states [Fig. 1(c)]. Our results and symmetry analysis show that the $K$-valley dark exciton decays into a left-handed chiral phonon and a right-handed photon, whereas the $K^{\prime}$-valley dark exciton decays into a right-handed chiral phonon and a 


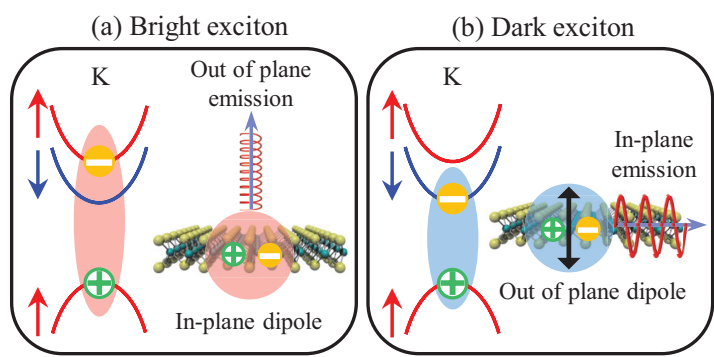

(c) Dark-exciton phonon replica

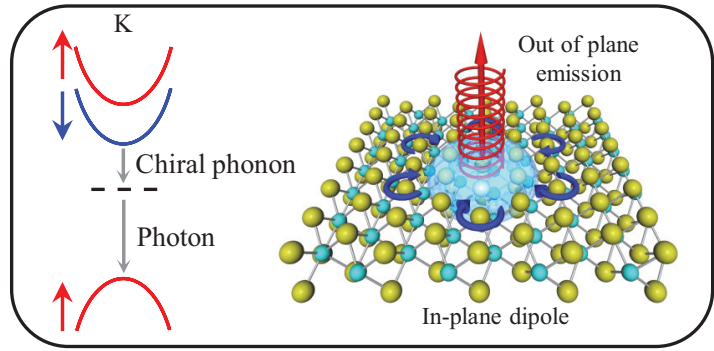

FIG. 1. (a)-(c) Band configurations, transition dipole, and optical emission of (a) bright exciton, (b) dark exciton, and (c) darkexciton chiral phonon replica at the $K$ valley in monolayer $\mathrm{WSe}_{2}$. The arrows denote the electron spin. A dark exciton can decay into a chiral phonon and a photon with opposite chirality.

left-handed photon. The replica PL intensity can be accounted for by first-principles calculations. Such valley-selective chiral phonon replicas can be utilized to identify the valley pseudospin of the dark excitonic states and explore the chiral exciton-phonon interactions.
We measure the photoluminescence (PL) from ultraclean monolayer $\mathrm{WSe}_{2}$ devices encapsulated by boron nitride on $\mathrm{Si} / \mathrm{SiO}_{2}$ substrates under continuous 532-nm laser excitation at temperature $T \sim 4 \mathrm{~K}$ [43]. Although the dark-state PL propagates in the IP directions, we can partially capture it with a wide-angle microscope objective [numerical aperture $(\mathrm{NA})=0.6]$ in the OP direction [16,28,43-47]. Figure 2(a) displays a gate-dependent PL map. The exceptional quality of our device allows us to observe a panoply of emission features, including the bright $A$ exciton $\left(A^{0}\right)$ and trions $\left(A_{1}^{-}\right.$, $\left.A_{2}^{-}, A^{+}\right)$, and dark exciton $\left(D^{0}\right)$ and trions $\left(D^{-}, D^{+}\right)[16,17]$. Notably, a PL peak emerges at $\sim 21.4 \mathrm{meV}$ below each of the $D^{0}, D^{-}$, and $D^{+}$peaks [Figs. 2(a) and 2(b)]. We denote them as $D_{p}^{0}, D_{p}^{-}$, and $D_{p}^{+}$, respectively. We have fit the spectra with multiple Lorentzian functions and extracted their PL intensity and energy. Both their intensity and energy shift show a similar gate dependence as the $D^{0}, D^{-}$, and $D^{+}$ peaks [Figs. 2(c) and 2(d)]. We can visualize the parallel gate dependence in a second-derivative PL map $\left(d^{2} I / d E^{2}\right)$, where the replica features are much sharpened [Fig. 2(e)].

The $D_{p}^{0}, D_{p}^{-}$, and $D_{p}^{+}$peaks exhibit almost the same $g$ factors as the $D^{0}, D^{-}$, and $D^{+}$peaks when we measure their Zeeman effect under an out-of-plane magnetic field $(B)$. The magnetic field can induce different band gaps in the two valleys [48-51]. The difference between the two valley gaps is the valley Zeeman splitting energy $\Delta E=g \mu_{\mathrm{B}} B$, where $g$ is the effective $g$-factor and $\mu_{\mathrm{B}}=57.88 \mu \mathrm{eV} / \mathrm{T}$ is the Bohr magneton. Figures 3(a)-3(f) display the $B$-dependent secondderivative PL maps (the raw data is in the Supplemental Material [43]). From the linear Zeeman shift we can extract (a)

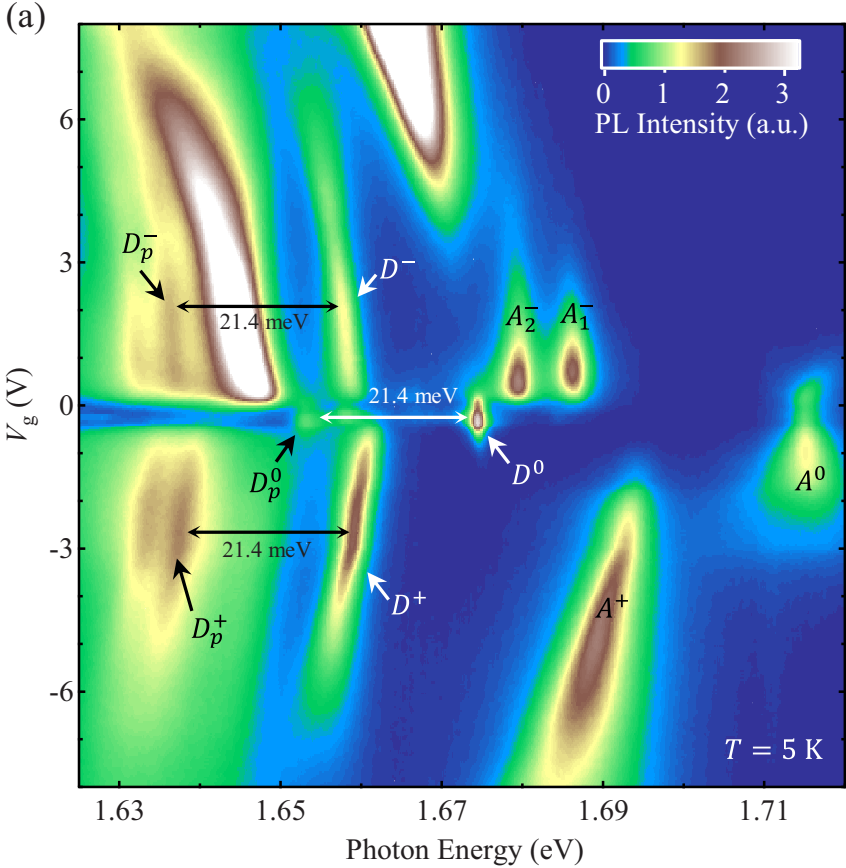

(b)
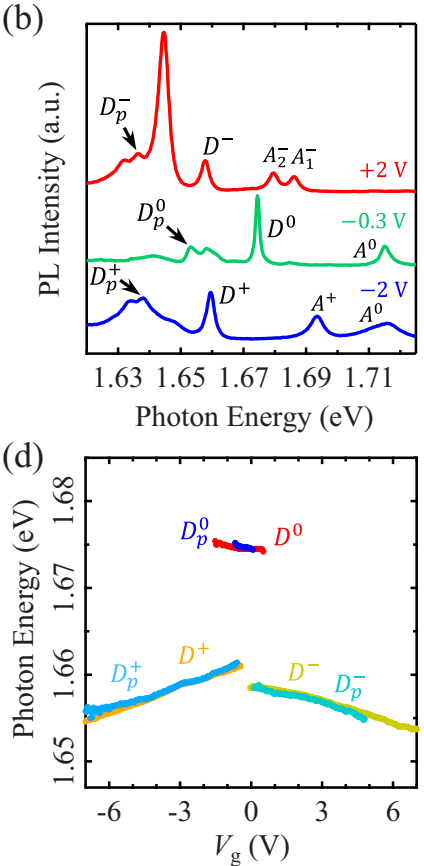

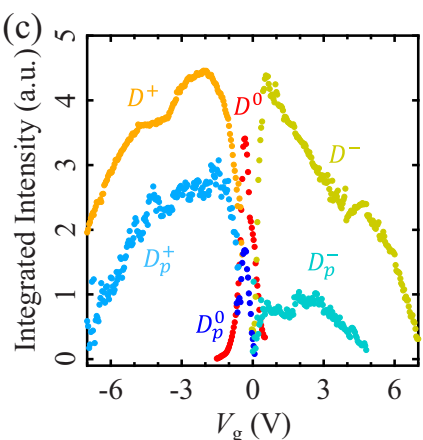

(e)

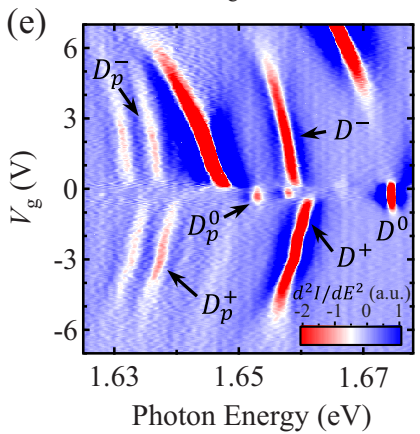

FIG. 2. (a) Gate-dependent photoluminescence (PL) map of a monolayer $\mathrm{WSe}_{2}$ device encapsulated by boron nitride. We denote the bright excitonic states $\left(A^{0}, A^{+}, A_{1}^{-}, A_{2}^{-}\right)$, dark excitonic states $\left(D^{0}, D^{-}, D^{+}\right)$, and dark states phonon replicas $\left(D_{p}^{0}, D_{p}^{-}, D_{p}^{+}\right)$. (b) The cross-cut PL spectra at the charge neutrality point (gate voltage $\left.V_{g}=-0.3 \mathrm{~V}\right)$, electron side $\left(V_{g}=2 \mathrm{~V}\right.$ ), and hole side $\left(V_{g}=-2 \mathrm{~V}\right)$. (c) PL intensity and (d) PL photon energy of the dark excitonic states and replicas as a function of gate voltage. The replica energy is upshifted for $21.4 \mathrm{meV}$ for comparison. (e) The second energy derivative $\left(d^{2} I / d E^{2}\right)$ of the PL map in (a). 

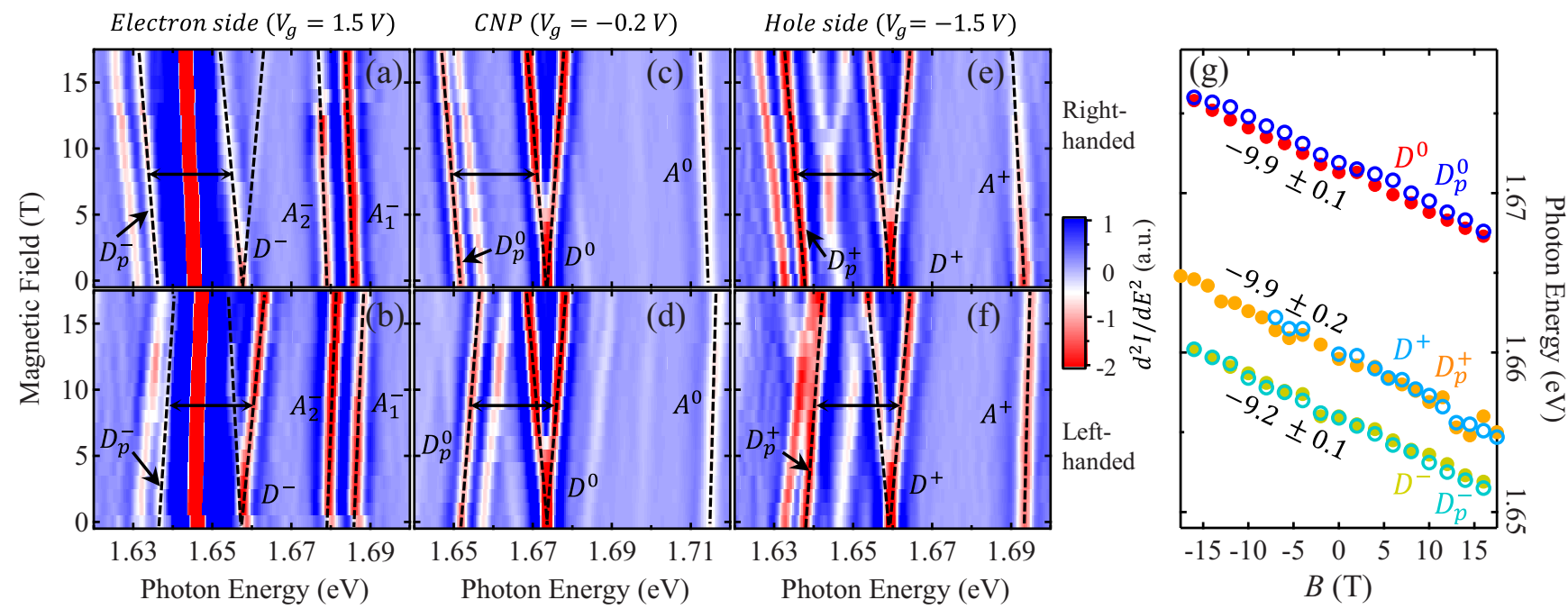

FIG. 3. (a)-(f) Magnetic-field-dependent second-derivative PL map $\left(d^{2} I / d E^{2}\right)$ of monolayer WSe $\mathrm{W}_{2}$ on the electron side [(a), (b) gate voltage $V_{g}=1.5 \mathrm{~V}$ ], near the charge neutrality point (CNP) [(c), (d) $V_{g}=-0.2 \mathrm{~V}$ ], and on the hole side [(e), (f) $\left.V_{g}=-1.5 \mathrm{~V}\right]$. We excite the sample with a linearly polarized 532-nm laser and collect the PL with right-handed (top row) or left-handed helicity (bottom row). (g) The Zeeman energy shift of one branch of the dark states and their replicas. The replica energy is upshifted for $21.4 \mathrm{meV}$ for comparison. The Zeeman-splitting $g$-factors from linear fits are denoted.

the $g$-factors. The $D_{p}^{0}, D_{p}^{-}$, and $D_{p}^{+}$peaks and the $D^{0}, D^{-}$, and $D^{+}$peaks have almost the same $g$-factors between -9.2 and -9.9 [Fig. 3(h)].

The parallel gate dependence and same $g$-factors strongly indicate that $D_{p}^{0}, D_{p}^{-}$, and $D_{p}^{+}$are the replicas of the $D^{0}$, $D^{-}$, and $D^{+}$peaks. We assign the $D_{p}^{0}, D_{p}^{-}$, and $D_{p}^{+}$peaks as the $E^{\prime \prime}$-mode phonon replicas because the redshift energy $\left(\sim 21.4 \mathrm{meV}\right.$ ) matches the $E^{\prime \prime}$ optical phonon energy in monolayer $\mathrm{WSe}_{2}$ [40,52-55]. Similar $E^{\prime \prime}$ phonon replicas have been reported in quantum-dot excitons in monolayer $\mathrm{WSe}_{2}$ [40]. In our experiment, the $E^{\prime \prime}$ phonon replica is found only for the dark excitonic states, not for the bright excitonic states.

Although $D_{p}^{0}, D_{p}^{-}$, and $D_{p}^{+}$are the replicas of the dark states, they appear to follow the optical selection rules of the bright states. In the magneto-PL experiment for Fig. 3, we excite the sample with a linearly polarized laser and detect the PL with right- or left-handed helicity. Such measurements detect the dark states from both valleys because they emit linearly polarized light. But they detect the bright states only from one valley because the bright states emit right-handed (left-handed) light from the $K\left(K^{\prime}\right)$ valley. Correspondingly, in our PL maps the $D^{0}, D^{-}$, and $D^{+}$peaks are each split into two branches under a magnetic field, corresponding to the two valleys [Figs. 3(a)-3(f)]. But the bright states only show a Zeeman shift with no splitting, because we can only detect one valley. Remarkably, the three replica peaks exhibit the same behavior as the bright states-they also only show a Zeeman shift with no splitting. In the right-handed PL detection, they shift in parallel with the lower branch of the dark states; we only observe the phonon replicas from the $K$ valley. In the left-handed PL detection, they shift in parallel with the higher branch of the dark states; we only observe the phonon replicas from the $K^{\prime}$ valley. The helicity of the phonon replicas therefore tells us the valley pseudospin of the original dark states.
We have further obtained the transition dipole orientation of the phonon replicas. Tang et al. recently developed a special method to measure the dipole direction of the excitonic emission [17]. They deposit monolayer $\mathrm{WSe}_{2}$ on a planar GaSe waveguide, which collects the light emission in the IP directions from both the IP and OP dipoles in monolayer $\mathrm{WSe}_{2}$. By measuring the polarization of such emission, they can resolve the PL components from the IP and OP dipole [43]. We have extracted the exciton and replica PL intensity from their data and plot them as a function of polarization angle in Fig. 4. The dark and bright states exhibit perpendicular PL polarizations

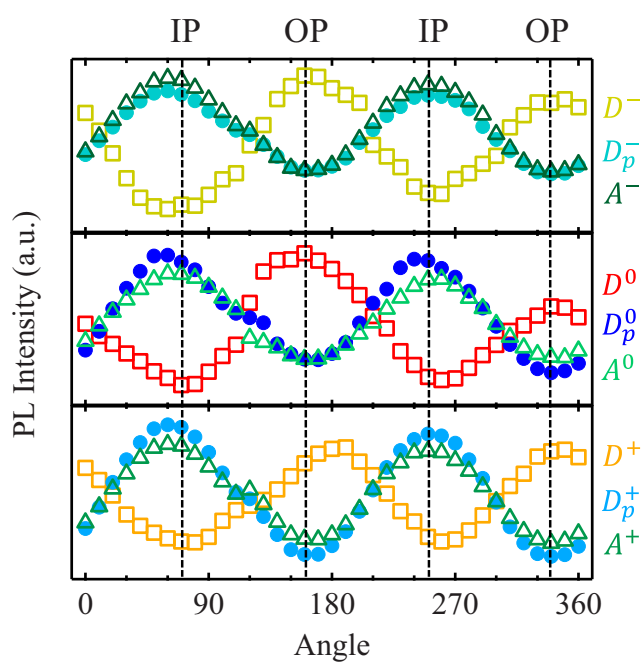

FIG. 4. The PL intensity of the bright excitonic states (triangle), dark excitonic states (square), and dark-state phonon replicas (dots) as a function of polarization angle in the in-plane collection geometry. The angles corresponding to in-plane (IP) and out-of-plane (OP) dipoles are denoted. 
TABLE I. Symmetry quantum numbers for the electronic bands $\left(c_{\uparrow}, \bar{c}_{\downarrow}, v_{\uparrow}\right)$ at the $K$ point and the $E^{\prime \prime}$ chiral phonons $\left(\Omega^{+}, \Omega^{-}\right)$at the zone center for monolayer $\mathrm{WSe}_{2}$.

\begin{tabular}{cccccc}
\hline \hline & $c_{\uparrow}$ & $\bar{c}_{\downarrow}$ & $v_{\uparrow}$ & $\Omega^{+}$ & $\Omega^{-}$ \\
\hline$\sigma_{h}$ & $i$ & $-i$ & $i$ & -1 & -1 \\
$C_{3}$ & $+\frac{1}{2}$ & $-\frac{1}{2}$ & $-\frac{1}{2}$ & +1 & -1 \\
\hline \hline
\end{tabular}

because they have OP and IP dipoles, respectively. Notably, the phonon replicas have the same polarization as the bright states, indicating that they are associated with the IP dipole.

The optical selection rules and the IP dipole of the phonon replicas can be explained by the symmetry of the electron and phonon states by group theory (see Supplemental Material for details [43]). The electronic states at the $K / K^{\prime}$ point possess the $C_{3 h}$ symmetry point group, including the OP mirror symmetry $\left(\hat{\sigma}_{h}\right)$ and IP threefold rotation symmetry $\left(\hat{C}_{3}\right)$. An eigenfunction $\psi$ transforms as $\hat{C}_{3} \psi=e^{-i \frac{2 \pi}{3} C_{3}} \psi$ and $\hat{\sigma}_{h} \psi=\sigma_{h} \psi$, where $C_{3}$ and $\sigma_{h}$ are the respective $\hat{C}_{3}$ and $\hat{\sigma}_{h}$ quantum numbers for $\psi$. Table I lists these quantum numbers for the spin-up and spin-down conduction bands $\left(c_{\uparrow}, \bar{c}_{\downarrow}\right)$ and the spin-up valence band $\left(v_{\uparrow}\right)$ at the $K$ point. The hat on $\bar{c}_{\downarrow}$ denotes that it is not purely spin-down, but contains a small spin-up component from coupling to a higher spin-up band by spin-orbit coupling $[49,56]$. Such spin mixing is necessary for the dark exciton to emit light. Under the symmetry operations, the $v-c$ interband transition matrices transform as $[56,57]$

$$
\begin{aligned}
\left\langle c\left|\hat{p}_{ \pm}\right| v\right\rangle & =\left\langle c\left|\hat{C}_{3}^{-1} \hat{C}_{3} \hat{p}_{ \pm} \hat{C}_{3}^{-1} \hat{C}_{3}\right| v\right\rangle \\
& =e^{i \frac{2 \pi}{3}\left[C_{3}(c)-C_{3}(v) \mp 1\right]}\left\langle c\left|\hat{p}_{ \pm}\right| v\right\rangle, \\
\left\langle c\left|\hat{p}_{ \pm}\right| v\right\rangle & =\left\langle c\left|\hat{\sigma}_{h}^{-1} \hat{\sigma}_{h} \hat{p}_{ \pm} \hat{\sigma}_{h}^{-1} \hat{\sigma}_{h}\right| v\right\rangle=\sigma_{h}^{*}(c) \sigma_{h}(v)\left\langle c\left|\hat{p}_{ \pm}\right| v\right\rangle, \\
\left\langle c\left|\hat{p}_{z}\right| v\right\rangle & =\left\langle c\left|\hat{C}_{3}^{-1} \hat{C}_{3} \hat{p}_{z} \hat{C}_{3}^{-1} \hat{C}_{3}\right| v\right\rangle=e^{i \frac{2 \pi}{3}\left[C_{3}(c)-C_{3}(v)\right]}\left\langle c\left|\hat{p}_{z}\right| v\right\rangle, \\
\left\langle c\left|\hat{p}_{z}\right| v\right\rangle & =\left\langle c\left|\hat{\sigma}_{h}^{-1} \hat{\sigma}_{h} \hat{p}_{z} \hat{\sigma}_{h}^{-1} \hat{\sigma}_{h}\right| v\right\rangle=-\sigma_{h}^{*}(c) \sigma_{h}(v)\left\langle c\left|\hat{p}_{z}\right| v\right\rangle .
\end{aligned}
$$

Here, the momentum operators $\hat{p}_{ \pm}=\hat{p}_{x} \pm i \hat{p}_{y}$ and $\hat{p}_{z}$ are associated with the IP chiral dipole and OP dipole, respectively. They transform as $\hat{C}_{3} \hat{p}_{ \pm} \hat{C}_{3}^{-1}=e^{\mp i \frac{2 \pi}{3}} \hat{p}_{ \pm}, \hat{\sigma}_{h} \hat{p}_{ \pm} \hat{\sigma}_{h}^{-1}=$ $\hat{p}_{ \pm}, \hat{C}_{3} \hat{p}_{z} \hat{C}_{3}^{-1}=\hat{p}_{z}$, and $\hat{\sigma}_{h} \hat{p}_{z} \hat{\sigma}_{h}^{-1}=-\hat{p}_{z}$. For a matrix element to be finite, the prefactor after the symmetry transformation must be one. From Table I, we can verify that only $\left\langle c_{\uparrow}\left|\hat{p}_{+}\right| v_{\uparrow}\right\rangle$ and $\left\langle\bar{c}_{\downarrow}\left|\hat{p}_{z}\right| v_{\uparrow}\right\rangle$ can be finite, whereas other transition matrix elements are all zero. Therefore, the bright and dark excitons in the $K$ valley are coupled exclusively to right-handed light and vertically polarized light, respectively [28,35-38].

When the atoms move due to the lattice vibration, the original states are no longer eigenstates. In particular, the electron-phonon coupling will renormalize the $\bar{c}_{\downarrow}$ band into

$$
\left|\overline{\bar{c}}_{\downarrow}\right\rangle=\left|\bar{c}_{\downarrow}\right\rangle+\frac{\left\langle c_{\uparrow}, \Omega\left|\hat{H}_{e p}\right| \bar{c}_{\downarrow}\right\rangle}{E_{\bar{c}_{\downarrow}}-E_{c_{\uparrow}}-\hbar \Omega}\left|c_{\uparrow}\right\rangle .
$$

Here, $|\Omega\rangle$ denotes an $E^{\prime \prime}$ phonon with frequency $\Omega$. In the chiral mode, the $\mathrm{W}$ atoms stay stationary and the Se atoms rotate counterclockwise or clockwise, giving rise to right-handed (a)

(b) $\mathrm{K}$

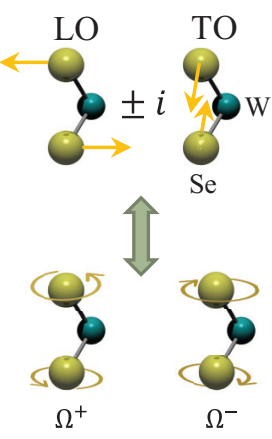

Chiral phonons

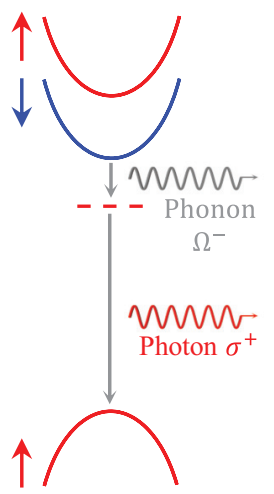

$\mathrm{K}^{\prime}$

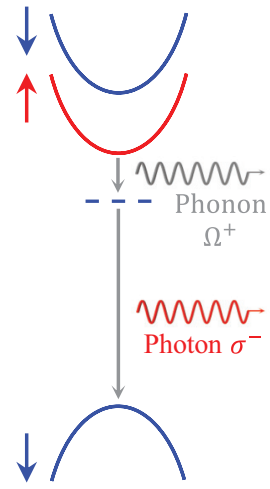

FIG. 5. (a) The configurations of the doubly degenerate zonecenter $E^{\prime \prime}$ phonons in monolayer $\mathrm{WSe}_{2}$. The $\mathrm{W}$ atoms are stationary and the Se atoms move laterally. The vibration can be decomposed into the LO and TO modes with linear Se atomic motion or lefthanded and right-handed chiral modes with rotational Se atomic motion. (b) The phonon-assisted radiative recombination of the dark exciton. The dark exciton can decay into a pair of phonon and photon with opposite chirality.

$\left(\Omega^{+}\right)$or left-handed $\left(\Omega^{-}\right)$phonons [Fig. 5(a)]. These phonons have odd mirror parity to mix the bright and dark states with opposite mirror parity. The chiral phonons also have threefold rotation symmetry with quantum numbers $C_{3}\left(\Omega^{ \pm}\right)= \pm 1$ (Table I). Upon a $\hat{C}_{3}$ rotation, the matrix element transforms as

$$
\left\langle c_{\uparrow}, \Omega\left|\hat{H}_{e p}\right| \bar{c}_{\downarrow}\right\rangle=e^{i \frac{2 \pi}{3}\left[1+C_{3}(\Omega)\right]}\left\langle c_{\uparrow}, \Omega\left|\hat{H}_{e p}\right| \bar{c}_{\downarrow}\right\rangle .
$$

The matrix element can only be finite for the left-handed phonon with $C_{3}\left(\Omega^{-}\right)=-1$. Therefore, the $K$-valley dark exciton only emits the left-handed chiral phonon.

The dark exciton can obtain oscillator strength from the bright exciton through the electron-phonon coupling $\hat{H}_{e p}$ and recombine through the $\overline{\bar{c}}_{\downarrow}-v_{\uparrow}$ transition by the electron-light interaction $\hat{H}_{e l}$. The Fermi's golden rule gives the transition rate [39]

$$
\begin{aligned}
P_{\overline{\bar{c}}_{\downarrow}-v_{\uparrow}} & \propto\left|\left\langle v_{\uparrow}, \omega, \Omega\left|\hat{H}_{e l}\right| \overline{\bar{c}}_{\downarrow}\right\rangle\right|^{2} \\
& \propto\left|\frac{\left\langle v_{\uparrow}, \omega, \Omega\left|\hat{H}_{e l}\right| c_{\uparrow}, \Omega\right\rangle\left\langle c_{\uparrow}, \Omega\left|\hat{H}_{e p}\right| \bar{c}_{\downarrow}\right\rangle}{E_{\bar{c}_{\downarrow}}-E_{c_{\uparrow}}-\hbar \Omega}\right|^{2} .
\end{aligned}
$$

Here, $|\omega\rangle$ denotes a photon with frequency $\omega$. $\left\langle v_{\uparrow}, \omega, \Omega\left|\hat{H}_{e l}\right| c_{\uparrow}, \Omega\right\rangle$ corresponds to the matrix element $\left\langle v_{\uparrow}|\hat{p}| c_{\uparrow}\right\rangle$ for the bright-exciton transition. Therefore, the chiral phonon replica follows the intensity and selection rules of the bright exciton.

By combining the phonon and photon selection rules, we conclude that the dark exciton emits a left-handed chiral phonon and a right-handed photon in the $K$ valley. By the time-reversal symmetry, it emits a right-handed chiral phonon and a left-handed photon in the $K^{\prime}$ valley [Fig. 5(b)]. These selection rules still hold even after we include the excitonic effect, because the exciton Hamiltonian has the same symmetry as the states at the $K / K^{\prime}$ point. Our experimental results are fully consistent with these selection rules. 
While the excitonic effect does not modify the selection rules, it can substantially enhance the intensity of the phonon replicas. In particular, the finite $k$-space extent of the exciton envelope functions allows coupling to phonons with finite momentum. Only one chiral component of these phonons will contribute to the transition, so the chiral phonon selection rules still hold. We have calculated the replica intensity in a full excitonic picture with the density functional theory. The calculated intensity ratio between the replica and dark exciton is $I_{D_{p}^{0}} / I_{D^{0}} \approx 0.02$. This is close to the experimental ratio $\left(I_{D_{p}^{0}} / I_{D^{0}} \approx 0.05\right)$ after we correct the different collection efficiency for the IP and OP emission in our setup (see Supplemental Material [43]). Our experiment shows different replica ratios for dark trions $\left(I_{D_{p}^{+}} / I_{D^{+}} \approx 0.02 ; I_{D_{p}^{-}} / I_{D^{-}} \approx\right.$ 0.07). This indicates that the coupling to the Fermi sea can affect the phonon replica emission.

In summary, we have observed chiral phonon replicas of dark excitons and trions in monolayer $\mathrm{WSe}_{2}$. The replicas exhibit rigorous chirality and valley optical selection rules, which allow us to access the dark-state valley pseudospin. The valley-selective replica emission can potentially be utilized to explore the valley dynamics of dark excitons and trions, such as to image their valley Hall effect. The replica process may also be used to generate phonons with selective chirality for the exploration of chiral phonon physics.

We thank Y. Tang, K. F. Mak, and J. Shan for sharing their dipole-resolved PL data with us. We thank D. Smirnov and $\mathrm{Z}$. Lu for assistance in the magneto-optical experiment. A portion of this work was performed at the National High Magnetic Field Laboratory, which is supported by the National Science Foundation Cooperative Agreement No. DMR1644779 and the State of Florida. Y.C.C. thanks C. T. Liang for assistance in the numerical calculation. Y.C.C. is supported by Ministry of Science and Technology (Taiwan) under Grant No. MOST 107-2112-M-001-032. K.W. and T.T. acknowledge support from the Elemental Strategy Initiative conducted by the MEXT, Japan and the CREST (JPMJCR15F3), JST.
[1] G. Wang, A. Chernikov, M. M. Glazov, T. F. Heinz, X. Marie, T. Amand, and B. Urbaszek, Colloquium: Excitons in atomically thin transition metal dichalcogenides, Rev. Mod. Phys. 90, 021001 (2018).

[2] K. F. Mak, C. Lee, J. Hone, J. Shan, and T. F. Heinz, Atomically Thin MoS: A New Direct-Gap Semiconductor, Phys. Rev. Lett. 105, 136805 (2010)

[3] A. Splendiani, L. Sun, Y. Zhang, T. Li, J. Kim, C.-Y. Chim, G. Galli, and F. Wang, Emerging photoluminescence in monolayer $\mathrm{MoS}_{2}$, Nano Lett. 10, 1271 (2010).

[4] D. Xiao, G.-B. Liu, W. Feng, X. Xu, and W. Yao, Coupled Spin and Valley Physics in Monolayers of $\mathrm{MoS}_{2}$ and Other Group-VI Dichalcogenides, Phys. Rev. Lett. 108, 196802 (2012).

[5] G.-B. Liu, W.-Y. Shan, Y. Yao, W. Yao, and D. Xiao, Three-band tight-binding model for monolayers of group-VIB transition metal dichalcogenides, Phys. Rev. B 88, 085433 (2013).

[6] K. Kośmider, J. W. González, and J. Fernández-Rossier, Large spin splitting in the conduction band of transition metal dichalcogenide monolayers, Phys. Rev. B 88, 245436 (2013).

[7] K. F. Mak and J. Shan, Photonics and optoelectronics of 2D semiconductor transition metal dichalcogenides, Nat. Photonics 10, 216 (2016).

[8] X. Xu, W. Yao, D. Xiao, and T. F. Heinz, Spin and pseudospins in layered transition metal dichalcogenides, Nat. Phys. 10, 343 (2014).

[9] A. Chernikov, T. C. Berkelbach, H. M. Hill, A. Rigosi, Y. Li, O. B. Aslan, D. R. Reichman, M. S. Hybertsen, and T. F. Heinz, Exciton Binding Energy and Nonhydrogenic Rydberg Series in Monolayer $\mathrm{WS}_{2}$, Phys. Rev. Lett. 113, 076802 (2014).

[10] K. He, N. Kumar, L. Zhao, Z. Wang, K. F. Mak, H. Zhao, and J. Shan, Tightly Bound Excitons in Monolayer $\mathrm{WSe}_{2}$, Phys. Rev. Lett. 113, 026803 (2014).

[11] A. V. Stier, N. P. Wilson, K. A. Velizhanin, J. Kono, X. Xu, and S. A. Crooker, Magnetooptics of Exciton Rydberg States in a Monolayer Semiconductor, Phys. Rev. Lett. 120, 057405 (2018).
[12] E. Liu, J. van Baren, T. Taniguchi, K. Watanabe, Y.-C. Chang, and C. H. Lui, Magnetophotoluminescence of exciton Rydberg states in monolayer $\mathrm{WSe}_{2}$, Phys. Rev. B 99, 205420 (2019).

[13] S.-Y. Chen, Z. Lu, T. Goldstein, J. Tong, A. Chaves, J. Kunstmann, L. S. R. Cavalcante, T. Woźniak, G. Seifert, D. R. Reichman et al., Luminescent emission of excited Rydberg excitons from monolayer $\mathrm{WSe}_{2}$, Nano Lett. 19, 2464 (2019).

[14] K. F. Mak, K. He, C. Lee, G. H. Lee, J. Hone, T. F. Heinz, and J. Shan, Tightly bound trions in monolayer $\mathrm{MoS}_{2}$, Nat. Mater. 12, 207 (2013).

[15] C. Robert, D. Lagarde, F. Cadiz, G. Wang, B. Lassagne, T. Amand, A. Balocchi, P. Renucci, S. Tongay, B. Urbaszek, and $\mathrm{X}$. Marie, Exciton radiative lifetime in transition metal dichalcogenide monolayers, Phys. Rev. B 93, 205423 (2016).

[16] E. Liu, J. van Baren, Z. Lu, M. M. Altaiary, T. Taniguchi, K. Watanabe, D. Smirnov, and C. H. Lui, Gate Tunable Dark Trions in Monolayer WSe $\mathrm{W}_{2}$, Phys. Rev. Lett. 123, 027401 (2019).

[17] Y. Tang, K. F. Mak, and J. Shan, Long valley lifetime of dark excitons in single-layer $\mathrm{WSe}_{2}$, Nat. Commun. 10, 4047 (2019).

[18] J. S. Ross, S. Wu, H. Yu, N. J. Ghimire, A. M. Jones, G. Aivazian, J. Yan, D. G. Mandrus, D. Xiao, W. Yao et al., Electrical control of neutral and charged excitons in a monolayer semiconductor, Nat. Commun. 4, 1474 (2013).

[19] D. K. Efimkin and A. H. MacDonald, Many-body theory of trion absorption features in two-dimensional semiconductors, Phys. Rev. B 95, 035417 (2017).

[20] Y.-C. Chang, S.-Y. Shiau, and M. Combescot, Crossover from trion-hole complex to exciton-polaron in $n$-doped twodimensional semiconductor quantum wells, Phys. Rev. B 98, 235203 (2018).

[21] M. Sidler, P. Back, O. Cotlet, A. Srivastava, T. Fink, M. Kroner, E. Demler, and A. Imamoglu, Fermi polaron-polaritons in charge-tunable atomically thin semiconductors, Nat. Phys. 13, 255 (2017). 
[22] X.-X. Zhang, Y. You, S. Y. F. Zhao, and T. F. Heinz, Experimental Evidence for Dark Excitons in Monolayer $\mathrm{WSe}_{2}$, Phys. Rev. Lett. 115, 257403 (2015).

[23] J. P. Echeverry, B. Urbaszek, T. Amand, X. Marie, and I. C. Gerber, Splitting between bright and dark excitons in transition metal dichalcogenide monolayers, Phys. Rev. B 93, 121107(R) (2016).

[24] M. R. Molas, C. Faugeras, A. O. Slobodeniuk, K. Nogajewski, M. Bartos, D. M. Basko, and M. Potemski, Brightening of dark excitons in monolayers of semiconducting transition metal dichalcogenides, 2D Mater. 4, 021003 (2017).

[25] K.-D. Park, T. Jiang, G. Clark, X. Xu, and M. B. Raschke, Radiative control of dark excitons at room temperature by nano-optical antenna-tip Purcell effect, Nat. Nanotechnol. 13, 59 (2018).

[26] X.-X. Zhang, T. Cao, Z. Lu, Y.-C. Lin, F. Zhang, Y. Wang, Z. Li, J. C. Hone, J. A. Robinson, D. Smirnov et al., Magnetic brightening and control of dark excitons in monolayer $\mathrm{WSe}_{2}$, Nat. Nanotechnol. 12, 883 (2017).

[27] Y. Zhou, G. Scuri, D. S. Wild, A. A. High, A. Dibos, L. A. Jauregui, C. Shu, K. De Greve, K. Pistunova, A. Y. Joe et al., Probing dark excitons in atomically thin semiconductors via near-field coupling to surface plasmon polaritons, Nat. Nanotechnol. 12, 856 (2017).

[28] G. Wang, C. Robert, M. M. Glazov, F. Cadiz, E. Courtade, T. Amand, D. Lagarde, T. Taniguchi, K. Watanabe, B. Urbaszek, and X. Marie, In-Plane Propagation of Light in Transition Metal Dichalcogenide Monolayers: Optical Selection Rules, Phys. Rev. Lett. 119, 047401 (2017).

[29] Z. Ye, T. Cao, K. O'Brien, H. Zhu, X. Yin, Y. Wang, S. G. Louie, and X. Zhang, Probing excitonic dark states in single-layer tungsten disulphide, Nature (London) 513, 214 (2014).

[30] C. Robert, T. Amand, F. Cadiz, D. Lagarde, E. Courtade, M. Manca, T. Taniguchi, K. Watanabe, B. Urbaszek, and X. Marie, Fine structure and lifetime of dark excitons in transition metal dichalcogenide monolayers, Phys. Rev. B 96, 155423 (2017).

[31] M. R. Molas, A. O. Slobodeniuk, T. Kazimierczuk, K. Nogajewski, M. Bartos, P. Kapuściński, K. Oreszczuk, K. Watanabe, T. Taniguchi, C. Faugeras, P. Kossacki, D. M. Basko, and M. Potemski, Probing and Manipulating Valley Coherence of Dark Excitons in Monolayer WSe ${ }_{2}$, Phys. Rev. Lett. 123, 096803 (2019).

[32] C. Monique, C. Roland, and D. François, Bose-Einstein condensation and indirect excitons: A review, Rep. Prog. Phys. 80, 066501 (2017).

[33] M. Combescot and M. N. Leuenberger, General argument supporting Bose-Einstein condensate of dark excitons in single and double quantum wells, Solid State Commun. 149, 567 (2009).

[34] M. Combescot, O. Betbeder-Matibet, and R. Combescot, BoseEinstein Condensation in Semiconductors: The Key Role of Dark Excitons, Phys. Rev. Lett. 99, 176403 (2007).

[35] J. A. Schuller, S. Karaveli, T. Schiros, K. He, S. Yang, I. Kymissis, J. Shan, and R. Zia, Orientation of luminescent excitons in layered nanomaterials, Nat. Nanotechnol. 8, 271 (2013).

[36] T. Cao, G. Wang, W. Han, H. Ye, C. Zhu, J. Shi, Q. Niu, P. Tan, E. Wang, B. Liu et al., Valley-selective circular dichroism of monolayer molybdenum disulphide, Nat. Commun. 3, 887 (2012).

[37] K. F. Mak, K. He, J. Shan, and T. F. Heinz, Control of valley polarization in monolayer $\mathrm{MoS}_{2}$ by optical helicity, Nat. Nanotechnol. 7, 494 (2012).

[38] H. Zeng, J. Dai, W. Yao, D. Xiao, and X. Cui, Valley polarization in $\mathrm{MoS}_{2}$ monolayers by optical pumping, Nat. Nanotechnol. 7, 490 (2012).

[39] Z. Li, T. Wang, C. Jin, Z. Lu, Z. Lian, Y. Meng, M. Blei, S. Gao, T. Taniguchi, K. Watanabe et al., Emerging photoluminescence from the dark-exciton phonon replica in monolayer $\mathrm{WSe}_{2}$, Nat. Commun. 10, 2469 (2019).

[40] X. Chen, X. Lu, S. Dubey, Q. Yao, S. Liu, X. Wang, Q. Xiong, L. Zhang, and A. Srivastava, Entanglement of single-photons and chiral phonons in atomically thin $\mathrm{WSe}_{2}$, Nat. Phys. 15, 221 (2019).

[41] H. Zhu, J. Yi, M.-Y. Li, J. Xiao, L. Zhang, C.-W. Yang, R. A. Kaindl, L.-J. Li, Y. Wang, and X. Zhang, Observation of chiral phonons, Science 359, 579 (2018).

[42] L. Zhang and Q. Niu, Chiral Phonons at High-Symmetry Points in Monolayer Hexagonal Lattices, Phys. Rev. Lett. 115, 115502 (2015).

[43] See Supplemental Material at http://link.aps.org/supplemental/ 10.1103/PhysRevResearch.1.032007 for a detailed description of experimental conditions, additional data, and calculations.

[44] M. Barbone, A. R. P. Montblanch, D. M. Kara, C. PalaciosBerraquero, A. R. Cadore, D. De Fazio, B. Pingault, E. Mostaani, H. Li, B. Chen et al., Charge-tuneable biexciton complexes in monolayer $\mathrm{WSe}_{2}$, Nat. Commun. 9, 3721 (2018).

[45] S.-Y. Chen, T. Goldstein, T. Taniguchi, K. Watanabe, and J. Yan, Coulomb-bound four- and five-particle intervalley states in an atomically-thin semiconductor, Nat. Commun. 9, 3717 (2018).

[46] Z. Li, T. Wang, Z. Lu, C. Jin, Y. Chen, Y. Meng, Z. Lian, T. Taniguchi, K. Watanabe, S. Zhang et al., Revealing the biexciton and trion-exciton complexes in $\mathrm{BN}$ encapsulated $\mathrm{WSe}_{2}$, Nat. Commun. 9, 3719 (2018).

[47] Z. Ye, L. Waldecker, E. Y. Ma, D. Rhodes, A. Antony, B. Kim, X.-X. Zhang, M. Deng, Y. Jiang, Z. Lu et al., Efficient generation of neutral and charged biexcitons in encapsulated $\mathrm{WSe}_{2}$ monolayers, Nat. Commun. 9, 3718 (2018).

[48] A. Srivastava, M. Sidler, A. V. Allain, D. S. Lembke, A. Kis, and A. Imamoğlu, Valley Zeeman effect in elementary optical excitations of monolayer $\mathrm{WSe}_{2}$, Nat. Phys. 11, 141 (2015).

[49] G. Aivazian, Z. Gong, A. M. Jones, R.-L. Chu, J. Yan, D. G. Mandrus, C. Zhang, D. Cobden, W. Yao, and X. Xu, Magnetic control of valley pseudospin in monolayer $\mathrm{WSe}_{2}$, Nat. Phys. 11, 148 (2015).

[50] Y. Li, J. Ludwig, T. Low, A. Chernikov, X. Cui, G. Arefe, Y. D. Kim, A. M. van der Zande, A. Rigosi, H. M. Hill, S. H. Kim, J. Hone, Z. Li, D. Smirnov, and T. F. Heinz, Valley Splitting and Polarization by the Zeeman Effect in Monolayer $\mathrm{MoSe}_{2}$, Phys. Rev. Lett. 113, 266804 (2014).

[51] D. MacNeill, C. Heikes, K. F. Mak, Z. Anderson, A Kormányos, V. Zólyomi, J. Park, and D. C. Ralph, Breaking of Valley Degeneracy by Magnetic Field in Monolayer $\mathrm{MoSe}_{2}$, Phys. Rev. Lett. 114, 037401 (2015). 
[52] X. Luo, Y. Zhao, J. Zhang, M. Toh, C. Kloc, Q. Xiong, and S. Y. Quek, Effects of lower symmetry and dimensionality on Raman spectra in two-dimensional WSe $\mathrm{W}_{2}$, Phys. Rev. B 88, 195313 (2013).

[53] S. Kim, K. Kim, J.-U. Lee, and H. Cheong, Excitonic resonance effects and Davydov splitting in circularly polarized Raman spectra of few-layer $\mathrm{WSe}_{2}$, 2D Mater. 4, 045002 (2017).

[54] Y. Song and H. Dery, Transport Theory of Monolayer Transition-Metal Dichalcogenides through Symmetry, Phys. Rev. Lett. 111, 026601 (2013).
[55] H. Dery and Y. Song, Polarization analysis of excitons in monolayer and bilayer transition-metal dichalcogenides, Phys. Rev. B 92, 125431 (2015)

[56] G.-B. Liu, D. Xiao, Y. Yao, X. Xu, and W. Yao, Electronic structures and theoretical modelling of two-dimensional groupVIB transition metal dichalcogenides, Chem. Soc. Rev. 44, 2643 (2015).

[57] H. Yu, G.-B. Liu, and W. Yao, Brightened spin-triplet interlayer excitons and optical selection rules in van der Waals heterobilayers, 2D Mater. 5, 035021 (2018). 